\title{
Mass food challenges in a vacant COVID-19 stepdown facility: exceptional opportunity provides a model for the future
}

\author{
Aideen Byrne ${ }^{1}$, Juan Trujillo ${ }^{2}$, John Fitzsimons ${ }^{1}$, Muhammad Tariq ${ }^{3}$, Robert Ghent ${ }^{1}$, \\ Cathryn O'Carroll ${ }^{1}$, David Coghlan ${ }^{1}$, and Jonathan Hourihane ${ }^{4}$ \\ ${ }^{1}$ Children's Health Ireland \\ ${ }^{2}$ Cork University Hospital \\ ${ }^{3}$ Midland Regional Hospital Portlaoise \\ ${ }^{4}$ Royal College of Surgeons in Ireland
}

April 23, 2021

\begin{abstract}
Background: Internationally, the COVID-19 pandemic severely curtailed access to hospital facilities for those awaiting elective/semi elective procedures. For allergic children in Ireland, already waiting up to 4yr for an elective oral food challenge (OFC), the restrictions signified indefinite delay. At the time of the initiative there were approx 900 children on the Chidren's Health Ireland(CHI) waiting list. In July 2020, a project was facilitated by short term(6wk) access to an empty COVID stepdown facility built, in a hotel conference centre, commandeered by the Health Service Executive Ireland(HSE). The aim was to the achieve rapid rollout of an off-site OFC service, delivering high throughput of long waiting patients, while aligning with hospital existing policies and quality standards, international allergy guidelines and national social distancing standards. Methods: The working group engaged key stakeholders to rapidly develop an offsite OFC facility. Consultant Paediatric Allergists, Consultant Paediatricians, trainees and Allergy Clinical Nurse Specialists were seconded from other duties. The facility was already equipped with hospital beds, bedside monitors(BP, Pulse, Oxygen saturation) bedside oxygen. All medication and supplies had to be brought from the base hospital. Daily onsite consultant anaesthetic cover was resourced and a resuscitation room equipped. Standardised food challenge protocols were created. Access to onsite hotel chef facilitated food preparation. A risk register was established. Results: After 6wks planning, the remote centre became operational on 7/9/20, with the capacity of $27 \mathrm{OFC} /$ day. 474 challenges were commenced, 465 (98\%) were completed, 9(2\%) were inconclusive. 135(29.03\%) OFC were positive, $25(5 \%)$ causing anaphylaxis. No child required advanced airway intervention. 8 children were transferred to the base hospital. The CHI allergy waiting list was reduced by almost $60 \%$ in only 24 days. Conclusions: OFCs remain a vital tool in the care of allergic children, with their cost saving and quality of life benefits negatively affected by delay in their delivery. This project has shown it is possible to have huge impacts on a waiting list efficiently, effectively and safely with good planning and staff buy in - even in a pandemic. Adoption of new, flexible and efficient models of service delivery will be important for healthcare delivery in the post-COVID-19 era.
\end{abstract}

\section{Hosted file}

Byrne_et_al_PAI0_2021.04.12.pdf available at https://authorea.com/users/408952/articles/ 519278-mass-food-challenges-in-a-vacant-covid-19-stepdown-facility-exceptionalopportunity-provides-a-model-for-the-future 